\title{
A Literature Review on Current Evidence of Immune Checkpoint Inhibitors in Advanced Non-Small Cell Lung Cancer
}

\author{
Vineet Kwatra ${ }^{1}$, Jose Roca ${ }^{2}$, Sophie Merrick ${ }^{1}$, Ruhe Chowdhury ${ }^{1}$, Eleni Karapanagiotou ${ }^{1}$ and Rohit Lal ${ }^{1}$ \\ ${ }^{1}$ Guy's and St Thomas' NHS Foundation Trust, London, United Kingdom \\ ${ }^{2}$ Royal Marsden Hospital, London, United Kingdom \\ *Corresponding author: Vineet Kwatra, MBBS, Medical Oncology, Guy's and St Thomas' NHS Foundation \\ Trust, London, United Kingdom, Tel: +44-20-7188-7188, E-mail: Vineet.Kwatra@gstt.nhs.uk
}

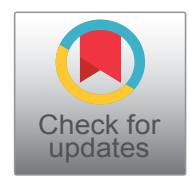

\begin{abstract}
Immune checkpoint inhibitors are changing the landscape in management of advanced non-small cell lung cancer. Programmed cell death protein-1 (PD-1) and cytotoxic T-lymphocytes antigen-4 (CTLA-4) are two important co-inhibitory receptors which can lead to suppression of T-cell function when bound to its ligands. Results from multiple large randomised phase 3 trials have shown a significant improvement in overall survival with durable response in selected group of patients following immune checkpoint blockage compared to second lines chemotherapy. Emerging evidences have shown promising responses to treatment in patients whose tumour has programmed cell death ligand -1 (PD-L1) $\geq 50 \%$ expression compared to standard first line platinum-based chemotherapy. Combination therapies of immune checkpoint inhibitors with other agents including chemotherapy are underway. Here we summarize the results of immune checkpoint blockade trials when used as monotherapy and in combination with other agents in advanced non-small cell lung carcinoma.
\end{abstract}

\section{Keywords}

Non-small cell lung cancer, Immune checkpoint inhibitor, PD-1, PD-L1, Pembrolizumab, Nivolumab

\section{Introduction}

The incidence of lung cancer has risen steadily over the past century with 6 million new cases diagnosed in 2008 globally. It is the commonest cancer seen in men worldwide and fourth commonest cancer seen in women. Lung cancer, now is the leading cause of cancer related deaths across all cancer types with 1.38 million deaths recorded in 2008 (18.2\%) of total cancer deaths [1]. The overall prognosis remains poor at 5 years only
$25 \%$ of those with locally advanced disease survived and $4 \%$ with distant metastatic disease [2]. Non-small cell lung cancer (NSCLC) accounts for $~ 85 \%$ of all lung cancer which can be further classify according to their histological subtypes. These subtypes can be categorised into adenocarcinoma, squamous cell, large cell and poorly differentiated cell carcinoma.

Chemotherapy remains the backbone of treatment for the majority of patients with metastatic NSCLC in the first-line setting. It has been shown to prolong survival, improve quality-of-life, and controls disease related symptoms compared to best supportive care [3]. The choice can be any of the four combination of platinum containing doublets chemotherapy which includes cisplatin combined with paclitaxel, gemcitabine, or docetaxel and carboplatin and paclitaxel. There are no significant differences survival benefit among these groups however the trial didn't stratified according to histology subtypes [4]. Later, Phase 3 trials comparing cisplatin and gemcitabine with cisplatin and pemetrexed have shown survival differences based on histological subtype. Superior survival benefit was shown in patient with adenocarcinoma who received cisplatin and pemetrexed compared to cisplatin and gemcitabine (12.6 vs. 10.9 months) respectively. On the other hand, patients with squamous cell subtype, a survival benefit of $10.8 \mathrm{vs.} 9.4$ months was shown when comparing cisplatin and gemcitabine to cisplatin and pemetrexed respectively [5]. Many patients with metastatic NSCLC will benefit from first line chemotherapy but after a median of 3-6 months they will likely progress and require second line treatment.

Citation: Kwatra V, Roca J, Merrick S, Chowdhury R, Karapanagiotou E, et al. (2018) A Literature Review on Current Evidence of Immune Checkpoint Inhibitors in Advanced Non-Small Cell Lung Cancer. Int J Cancer Clin Res 5:090. doi.org/10.23937/2378-3419/1410090

Received: September 02, 2017; Accepted: April 16, 2018; Published: April 18, 2018

Copyright: (C) 2018 Kwatra V, et al. This is an open-access article distributed under the terms of the Creative Commons Attribution License, which permits unrestricted use, distribution, and reproduction in any medium, provided the original author and source are credited. 


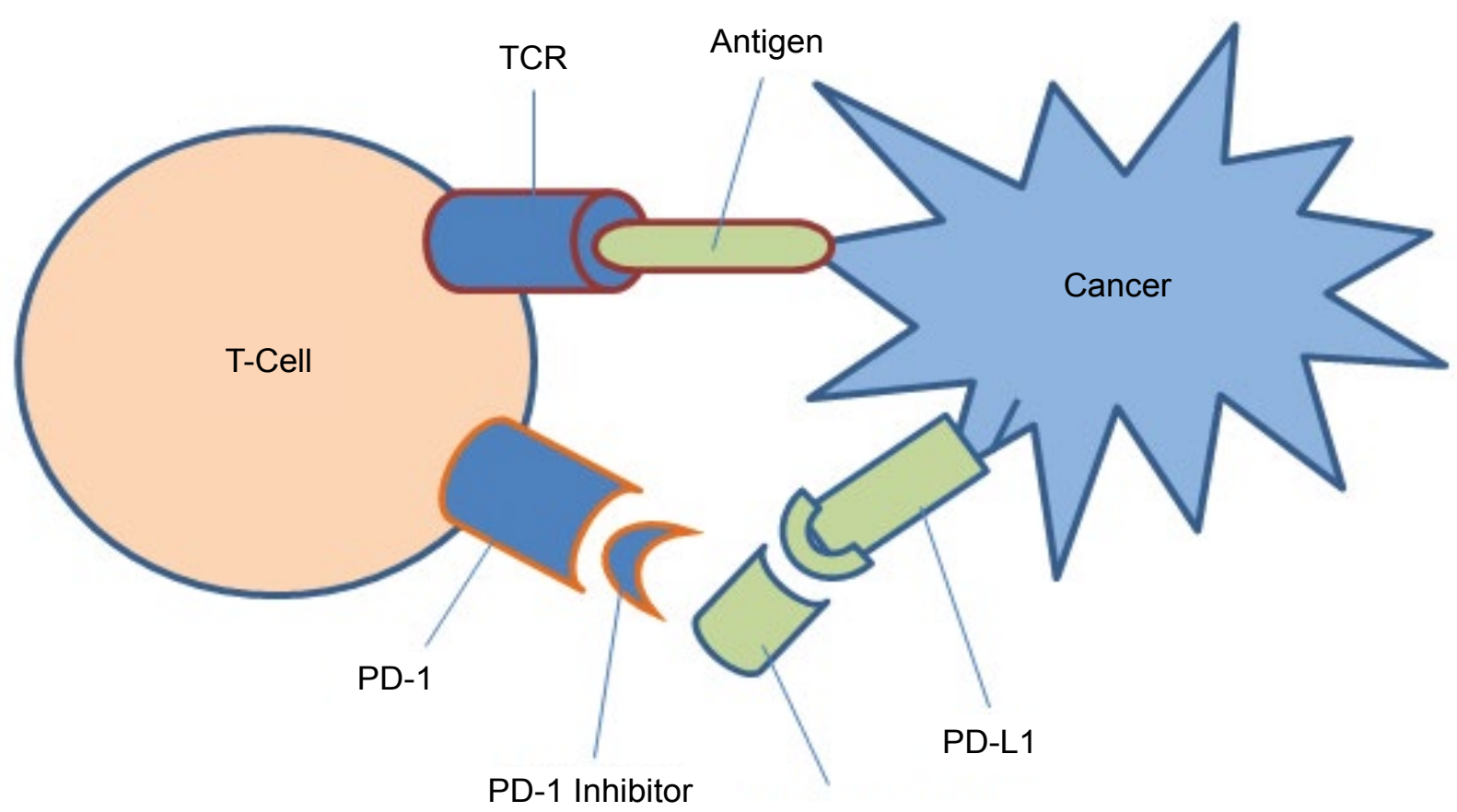

PD-L1 Inhibitor

Figure 1a: When PD-1 receptor on T-cell binds to PD-L1 receptor on tumour cell, its leads to downregulation of T-cell. However, immune checkpoint blockage with either PD-1 or PD-L1 inhibitor can interrupt this inhibitory signal and therefore leads to activation of T-cell toward tumour cells.

Over the past decade, there has been further identification of a subset of non-squamous NSCLC which can be further defined through the expression of driver mutations at a molecular level. These oncogenes drivers include the epidermal growth factor receptor (EGFR) mutation, echinoderm microtubule-associated protein-like 4 and anaplastic lymphoma kinase translocation (EML4-ALK) mutation, and Proton-Oncogene tyrosine protein kinase ROS 1 fusion. However, these oncogenes drivers are present in approximately $10-15 \%$ of patients with non-squamous NS$\mathrm{CLC}$. It is essential to identify whether an oncogene driver is present as there are several therapeutic targeted interventions with favourable survival outcomes for this selected group of patients. Testing for these driver mutations is now considered as routine for newly diagnosed cases of metastatic non-squamous NSCLC. A number of driver mutations have also been identified in squamous cell carcinoma but unfortunately there is no targeted intervention available outside clinical trial.

Despite advances in research and a better understanding of the molecular pathways in NSCLC, there has been a limited effective therapeutic option available for the majority of patients with advanced NSCLC. However, there has been a great interest in using immune checkpoint inhibitor for NSCLC following successful outcomes in melanoma. Here we will review the evidence of different immune checkpoint inhibitors in advanced NSCLC.

\section{Mechanism of Immune Checkpoint Blockage}

\section{PD-1 and PD-L1}

Programmed cell death protein 1 (PD-1) is a trans- membrane protein receptor that is expressed on the surfaces of T-cells, B-cells macrophages and dendritic cells. Its ligand, programmed cell death ligands 1 and 2 (PD-L1 and PD-L2; also known as B7-H1 and B7-DC respectively) belongs to immune checkpoint proteins that act as co-inhibitory factors. In normal circumstances, this interaction plays an important role in maintaining immune homeostasis including downregulation of the T-cell which promotes self-tolerance and protects against autoimmunity [6]. PD-L1 can be over expressed (20-50\%) in various types of malignancies including melanoma, renal cell carcinoma and colorectal cancer [7]. In NSCLC, PD-L1 is expressed in $50-60 \%$ of cases [8]. Once activated, it leads to suppression of cytotoxic T-cell proliferation and an inhibited tumour microenvironment. These anergic T-cells are known as tumour-infiltrating lymphocytes (TILS). Figure 1a illustrates the basic interaction between T-cell and cancer cell.

Recently, monoclonal antibodies against PD-1 and PD-L1 have been developed. The PD-1 blockage acts by enhancing the cytotoxic T-cell in tissues and TILs in tumour microenvironment resulting in upregulation and proliferation of intratumoral immune response against cancer. As the PD-1 receptors are also expressed on other non-T-cells, the PD-1 blockage can potentially exert on macrophages as well as B-cells to initiate antibody production [9].

\section{CTLA-4}

Cytotoxic T-lymphocyte-associated protein 4 (CTLA4 ) is a protein receptor found mainly on T-cells. It transmits an inhibitory signal to $T$ cells following recognition 


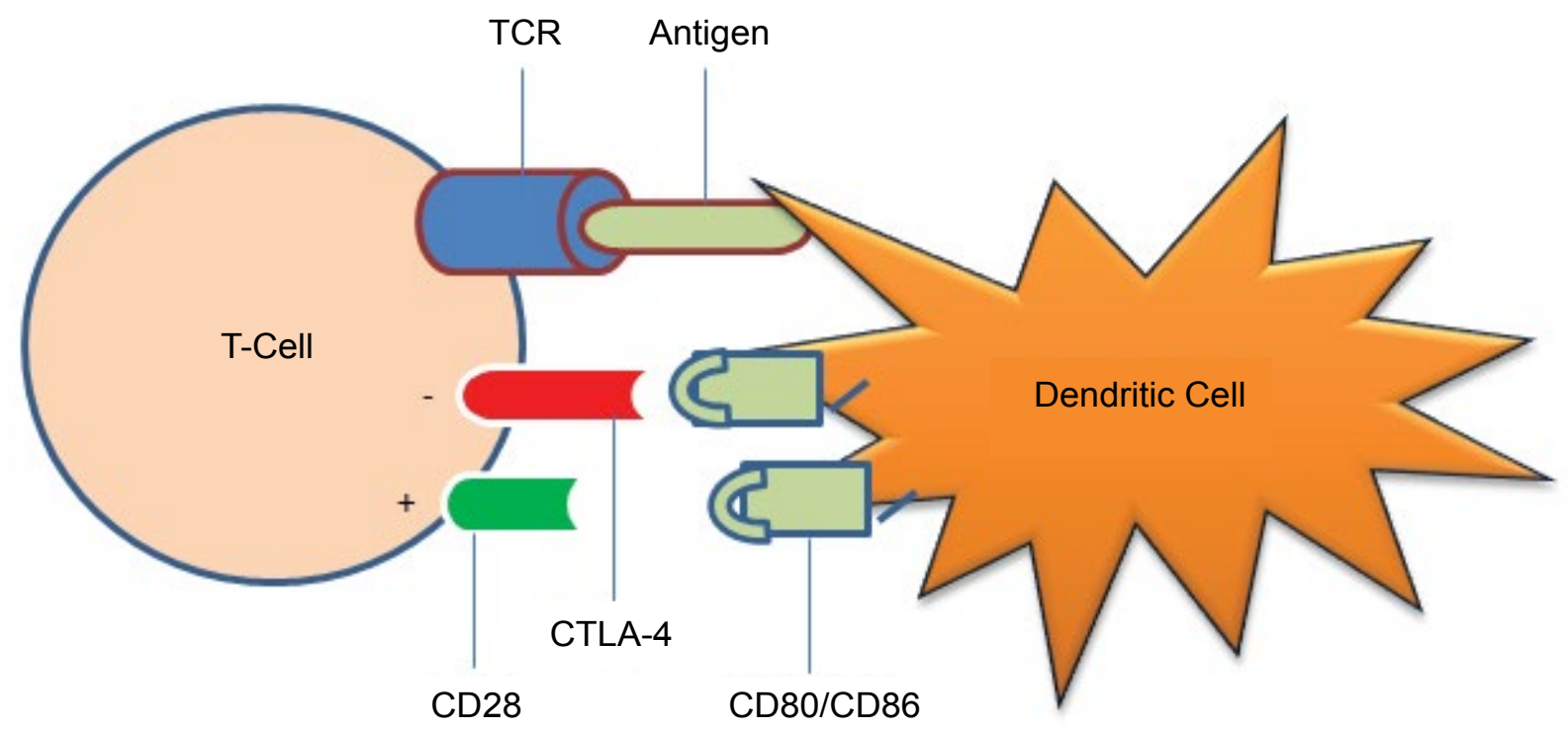

Figure 1b: T-cell activation is initiated by the interaction between CD28 receptors and CD80/CD86 on antigen presenting cell or dendritic cell. CTLA-4 receptor expressed on T-cell has higher affinity and inhibitory signal when binds to CD80/CD86 leading to T-cell inactivation.

between T-cell receptors (TCR) and peptides from antigen presenting cells (APCs). In contrast CD28, which is homologous to CTLA-4, is a T-cell costimulatory protein leading to proliferation of T cells. Both CTLA-4 and CD28 share similar ligands (CD80 and CD86). It is believed that CTLA-4 has a much higher binding affinity than CD28, allowing it to outcompete CD28 for these ligands, leading to an inhibitory signal to the T cell (Figure 1b) [10-11]. Ipilimumab and tremelimumab are two examples of fully humanized CTLA-4 antibodies which block the co-inhibitory signals between CTLA-4 receptor and its ligands and promote antitumour activity [12-13].

\section{PD-1 Inhibitors in Advanced NSCLC}

\section{Pembrolizumab}

Keynote-010, a randomised phase $2 / 3$ open-label controlled trial, compared pembrolizumab to docetaxel in previously treated PD-L1-positive metastatic NSCLC. The PD-L1 expressions are required to be $\geq 1 \%$ preferably on a new tissue biopsy. 1034 patients were randomly assigned (1:1:1) to received pembrolizumab $2 \mathrm{mg} / \mathrm{kg}$, pembrolizumab $10 \mathrm{mg} / \mathrm{kg}$, or docetaxel $75 \mathrm{mg} / \mathrm{m}^{2}$ every 3 weeks. The primary endpoints were overall survival (OS) and progression-free survival (PFS) both in the total population and in patients with PD-L1 expression $\geq 50 \%$. The median OS was 10.4 months with pembrolizumab $2 \mathrm{mg} / \mathrm{kg}, 12.7$ months with pembrolizumab $10 \mathrm{mg} / \mathrm{kg}$ and 8.5 months for docetaxel. Overall survival (OS) was significantly longer for pembrolizumab at $2 \mathrm{mg} / \mathrm{kg}$ and $10 \mathrm{mg} / \mathrm{kg}$ compared to docetaxel with hazard ratio (HR) of 0.71 and 0.61 respectively [14].

The survival benefit in both of the pembrolizumab arms was even greater in the patients who expressed $\geq 50 \%$ PD-L1. The median OS with pembrolizumab 2 $\mathrm{mg} / \mathrm{kg}$ compared to docetaxel was 14.9 months vs. 8.2 months; (HR 0.54, 95\%; confidence interval (Cl) 0.38$0.77 ; p=0.0002$ ) and pembrolizumab $10 \mathrm{mg} / \mathrm{kg}$ compared to docetaxel was 17.3 months vs. 8.2 months; (HR $0.55, \mathrm{Cl} 0.36-0.70 ; \mathrm{p}<0.001)$. The PFS in this subset was also significantly longer in both pembrolizumab arms compared to docetaxel, with median of 5.0 months vs. 5.2 months vs. 4.1 months respectively. The RR in total population of pembrolizumab $2 \mathrm{mg} / \mathrm{kg}$, pembrolizumab $10 \mathrm{mg} / \mathrm{kg}$, and docetaxel were $18 \%, 18 \%$, and $9 \%$ respectively. However in the population with PD-L1 expression $\geq 50 \%$, the RR was $30 \%, 29 \%$ and $8 \%$ respectively [14].

The study highlighted the importance of biomarkers and careful patient selection with a significant survival benefit in patients with advanced NSCLC who had received $\geq 1$ systemic treatment particularly if their PD$\mathrm{L} 1$ expression was $\geq 50 \%$. Furthermore, pembrolizumab has been shown to be well tolerated with fewer grade 3-5 treatment-related toxicity in comparison to docetaxel.

Keynote- 024 is a randomised, open-label, phase 3 trial comparing pembrolizumab fixed dose of $200 \mathrm{mg}$ every 3 weeks with the investigator's choice of first-line chemotherapy for patients with advanced NSCLC and PD-L1 expression $\geq 50 \%$. The primary endpoint was PFS. The median PFS was 10.3 months in the pembrolizumab arm versus 6.0 months in the chemotherapy arm (HR 0.50; $95 \% \mathrm{Cl} 0.37$ to $0.68 ; \mathrm{p}<0.001)$. The median OS based on 25.2 months follow up in pembrolizumab arm was 30.2 months versus 14.2 months in chemotherapy arm representing $37 \%$ risk reduction $(\mathrm{HR} 0.63 ; 95 \% \mathrm{Cl} 0.47-086 ; \mathrm{p}=$ 0.002 ). The ORR was $45.5 \%$ in pembrolizumab arm versus $29.8 \%$ in chemotherapy arm. Grade 3-4 treatment-related toxicity was seen similarly in both arms [15].

Given the data from Keynote-010 and Keynote-024 (Table 1), FDA has approved pembrolizumab for ad- 
Table 1: Clinical trials assessing PD-1 or PD-L1 immune checkpoint inhibitors as monotherapy in NSCLC.

\begin{tabular}{|c|c|c|c|c|c|c|c|}
\hline Clinical Trials & \begin{tabular}{|l|} 
Lines of \\
Treatment
\end{tabular} & Phase & Sample Size & ORR (\%) & PFS (Months) & OS (Months) & AEs $\geq 3(\%)$ \\
\hline $\begin{array}{l}\text { KEYNOTE 10: } \\
\text { Pembrolizumab vs. } \\
\text { Docetaxel }\end{array}$ & $\geq 2$ & Two & 1034 & & & & \\
\hline *Total Population & & & & 18 vs. 18 vs. 9 & $\begin{array}{l}3.9 \text { vs. } 4.0 \text { vs. } \\
4.0\end{array}$ & 10.4 vs. 12.7 vs. 8.5 & 13 vs. 16 vs. 35 \\
\hline PD-L1 $\geq 50 \%$ & & & & 30 vs. 29 vs. 8 & $\begin{array}{l}5.0 \text { vs. } 5.2 \text { vs. } \\
4.1\end{array}$ & 14.9 vs. 17.3 vs. 8.2 & \\
\hline $\begin{array}{l}\text { KEYNOTE 024: } \\
\text { Pembrolizumab vs. } \\
\text { Chemotherapy }\end{array}$ & 1 & Three & 305 & 45.5 vs. 29.8 & 10.3 vs. 6.0 & $\begin{array}{l}24 \text { mths OS - } 51.5 \% \\
\text { vs. } 34.5 \%\end{array}$ & 21.4 vs. 20.7 \\
\hline \multicolumn{8}{|l|}{ PD-L1 $\geq 50 \%$} \\
\hline $\begin{array}{l}\text { Checkmate } 017 \text { (sq- } \\
\text { NSCLC): Nivolumab } \\
\text { vs. Docetaxel }\end{array}$ & $\geq 2$ & Three & 260 & 20 vs. 9 & 3.5 vs. 2.8 & 9.2 vs. 6.0 & 7 vs. 54 \\
\hline & & & & & & $\begin{array}{l}1 \text { yr OS - } 80.2 \% \text { vs. } \\
72.4 \%\end{array}$ & \\
\hline $\begin{array}{l}\text { Checkmate } 057 \\
\text { (nonsq-NSCLC): } \\
\text { Nivolumab vs. } \\
\text { Docetaxel }\end{array}$ & $\geq 2$ & Three & 582 & 19 vs. 12 & 2.3 vs. 4.2 & 12.2 vs. 9.4 & 10 vs. 54 \\
\hline & & & & & $\begin{array}{l}18 \text { mths OS: } \\
39 \% \text { vs. } 23 \%\end{array}$ & & \\
\hline $\begin{array}{l}\text { Checkmate 026: } \\
\text { Nivolumab vs. } \\
\text { Platinum-doublet }\end{array}$ & 1 & Three & 541 & 26 vs. 34 & 4.2 vs. 5.9 & 14.2 vs. 13.2 & 18 vs. 51 \\
\hline $\begin{array}{l}\text { OAK: Atezolizumab } \\
\text { vs. Docetaxel }\end{array}$ & $\geq 2$ & Three & 850 & & & & \\
\hline Total Population & & & & 14 vs. 13 & 2.8 vs. 4.0 & 13.8 vs. 9.6 & 15 vs. 43 \\
\hline PD-L1 $11 \%$ & & & & & & 15.7 vs. 10.3 & \\
\hline Undetectable PD-L1 & & & & & & 12.6 vs. 8.9 & \\
\hline $\begin{array}{l}\text { ATLANTIC: Single- } \\
\text { arm Durvalumab }\end{array}$ & $\geq 2$ & Two & & & & & 10.2 \\
\hline $\begin{array}{l}\text { Cohort } 2 \text { - PD-L1 } \geq \\
25 \%\end{array}$ & & & 149 & 16.4 & 3.3 & 10.9 & \\
\hline PD-L1 $\leq 25 \%$ & & & 94 & 7.5 & 1.9 & 9.3 & \\
\hline $\begin{array}{l}\text { Cohort } 3 \text { - PD-L1 } 1 \geq \\
90 \%\end{array}$ & & & 67 & 30.9 & 2.4 & NR & \\
\hline
\end{tabular}

ORR: Overall Response Rate; PFS: Progression Free Survival; OS: Overall Survival; AEs: Adverse Effects; NR: Not reached. "Pembrolizumab 2 mg/kg vs. Pembrolizumab $10 \mathrm{mg} / \mathrm{kg}$ vs. Docetaxel 75 mg/m².

vanced NSCLC whose tumour have high PD-L1 expression $\geq 50 \%$ with no EGFR or ALK genomic tumour aberration, and no prior systemic chemotherapy in first-line management setting. The approval has also expanded to include patients with advanced NSCLC whose tumours express PD-L1 expression $\geq 1 \%$ following disease progression after platinum-containing chemotherapy. Patients with EGFR and ALK genomic tumour aberration should have evidence of disease progression on approved standard treatment prior to receiving pembrolizumab.

The design of Keynote-024 was targeted towards a specific group of patients with higher PD-L1 expression without targetable molecular lesions (EGFR mutations or ALK rearrangements) which may lead to significant ORR and PFS. Therefore, an attempt has been made to answer the question whether a lower PD-L1 expression (i.e. $\leq 50 \%$ ) will have similar endpoints. This will be evaluated in an ongoing phase 3 study, Keynote-042, which compares pembrolizumab to platinum-based chemotherapy in advanced NSCLC patients. The patient will be randomised into either a fixed dose pembrolizumab 200 mg every three weeks or carboplation plus paclitaxel or carboplatin plus pemetrexed. The treatment will be stratified according to the level of PD-L1 expression i.e PD-L1 $\geq 50 \%$ versus PD-L1 1-49\%. The primary end point is OS in total population and in patients with PD-L1 $\geq$ $50 \%$ expression [16].

\section{Nivolumab}

Nivolumab is an IgG4 monoclonal antibody that blocks PD-1 receptors. Checkmate 017, a phase 3 trial of 272 patients with advanced squamous NSCLC who had failed platinum-based doublet chemotherapy was randomly assigned to either nivolumab $(3 \mathrm{mg} / \mathrm{kg}$ every 2 weeks) or docetaxel ( $75 \mathrm{mg} / \mathrm{m}^{2}$ every three weeks). The primary end-point was OS. The median OS was 9.2 months $(95 \% \mathrm{Cl}, 7.3$ to 13.3$)$ with nivolumab versus 6.0 
months $(95 \% \mathrm{Cl}, 5.1$ to 7.3$)$ with docetaxel. The oneyear survival rate was $42 \%$ versus $24 \%$, hazard ratio [HR] $0.59,95 \% \mathrm{Cl} 0.44-0.79)$. The absolute risk reduction was $41 \%$ with nivolumab. The ORR was higher with nivolumab (20\% versus $9 \%)$. However, the trial has shown that PD-L1 expression was neither prognostic nor predictive for patient with advanced squamous NSCLC. The negative results for this biomarker may possibly due to the use of archived tissues instead of re-biopsy at disease progression. Grade $\geq 3$ toxicities were less frequent with nivolumab when compared to docetaxel ( $7 \%$ versus $54 \%)$ [17].

Another randomized phase 3 trial, Checkmate 057, was similar to Checkmate 017 however; the target population had advanced non-squamous NSCLC. 582 patients who had failed platinum-based doublet chemotherapy were randomly assigned to nivolumab $(3 \mathrm{mg} /$ kg every 2 weeks) or docetaxel $\left(75 \mathrm{mg} / \mathrm{m}^{2}\right.$ every three weeks). The primary end-point was OS. The median OS was 12.2 months $(95 \% \mathrm{Cl}, 9.7$ to 15.0$)$ with nivolumab versus 9.4 months $(95 \% \mathrm{Cl}, 8.1$ to 10.7$)$ with docetaxel. The 18 -months OS rate was $39 \%$ with nivolumab versus $23 \%$ with docetaxel (HR $0.72,95 \% \mathrm{Cl} 0.60-0.88$ ). The ORR for nivolumab and docetaxel were $19 \%$ and $12 \%$, respectively. The median durations of response were 17 months and 6 months, respectively. However, in this trial, patients with any level of PD-L1 expression had a survival benefit compared to negative PD-L1 level. The stronger the expression of PD-L1, the higher the survival gain. The treatment related toxicity was similar to Checkmate 017 [18].

As nivolumab has shown survival benefit in second-line treatment for advanced NSCLC, it was compared to standard first-line platinum-doublet chemotherapy in the phase 3 Checkmate 026 trial. 541 patients with advanced NSCLC with $\geq 1 \%$ PD-L1 expression who were treatment naïve were randomly assigned to either nivolumab ( $3 \mathrm{mg} / \mathrm{kg}$ every two weeks) or any platinum-doublet chemotherapy. The primary endpoint was PFS and the secondary endpoint was OS and ORR. Interestingly, the results did not show a dramatic response in patient with $>5 \%$ PD-L1 expression, with median PFS in nivolumab arm of 4.2 months versus platinum doublet chemotherapy arm of 5.9 months. The one-year PFS rate was $24 \%$ versus $23 \%$ respectively; (HR 1.15 , $95 \% \mathrm{Cl}$ 0.91-1.45). The OS was also not prolonged with nivolumab compared to platinum-doublet chemotherapy with median OS of 14.2 months versus 13.2 months respectively; (HR 1.02, 95\% Cl 0.80-1.30). The ORR was $26 \%$ compared to $34 \%$ respectively. However the median duration of response was 12.1 months with nivolumab compared to 5.7 months with chemotherapy. This suggested a durable response to a selected group of patients in the nivolumab arm. Again the treatment related toxicities were similar; grade $\geq 3$ toxicity was $18 \%$ with nivolumab, compared to $51 \%$ with platinum doublet chemotherapy [19].
It is unclear why there are differences in OS between Keynote 024 and Checkmate 026 . One possibility could be due to different PD-L1 expression cut offs. Another possibility was the difference between timing of biopsy prior to treatment. Keynote 024 tested PD-L1 expression at diagnosis of metastases while Checkmate 026 performed the test on archived samples.

\section{PD-L1 in Advanced NSCLC}

\section{Atezolimumab}

Atezolimumab is an IgG1 antibody which blocks the PD-L1 receptor thereby activating the tumour infiltrating immune cells that express PD-L1. In an open label, single-arm phase $2 \mathrm{BIRCH}$ study which recruited $667 \mathrm{ad}$ vanced NSCLC patients into 3 cohorts [20]. There were 142 patients with treatment naïve NSCLC (cohort 1), 271 patients who has progressed after platinum-doublet chemotherapy (Cohort 2), and 254 patients who had $\geq 2$ previous lines of chemotherapy (Cohort 3 ). Only patients with whose tumour expressed PD-L1 at a higher IHC level were enrolled. Atezolizumab was administered at 1,200 mg IV every 3 weeks. The primary endpoint was ORR. The ORR in cohort 1 was 19\%, and $17 \%$ in both cohorts 2 and 3 in all level of PD-L1 expression. Six month OS was achieved by $79 \%, 80 \%$ and $75 \%$ in cohort 1 to 3 respectively. There was no unexpected toxicity compared to PD-1 inhibitors.

Another open label phase 2 randomised controlled trial (POPLAR) compared IV atezolizumab $1,200 \mathrm{mg}$ or docetaxel $75 \mathrm{mg} / \mathrm{m}^{2}$ once every 3 weeks in previously treated advanced NSCLC [21]. Patients were stratified according to PD-L1 expression. The primary endpoint was overall survival. The OS was 12.6 months $(95 \% \mathrm{Cl}$ 9.7-16.4) for atezolizumab versus 9.7 months (8.6-12.0) for docetaxel (HR 0.73 [95\% Cl 0.53-0.99]; $p=0.04$ ). Given above data has shown survival benefit, atezolizumab progressed to phase 3 clinical trial. The OAK study was a randomised, open label, phase 3 trial comparing atezolizumab to docetaxel in previously treated advanced NSCLC [22]. The primary endpoint was OS. 850 patients were randomly assigned to with atezolizumab or docetaxel. An OS benefit was seen with atezolizumab compared to docetaxel (median OS 13.8 months [95\% $\mathrm{Cl} 11.8-15.7$ ] versus 9.6 months [8.6-11.2]; HR 0.73 [95\% Cl 0.62-0.87], $p=0.0003$ ). The OS in PD-L1 $\geq 1 \%$ was 15.7 months with atezolizumab versus 10.3 months with docetaxel. However, patients with undetectable PD-L1 expression also demonstrated an OS benefit with atezolizumab in comparison to docetaxel, 12.6 months versus 8.9 months respectively. The survival benefit is not as great as in patients with high levels of PD-L1 expression. The survival benefit was also seen in other subgroups including histology (HR 0.73 for both squamous and non-squamous), brain metastasis (HR 0.54) and never smokers (HR 0.71). With these results, the FDA has now approved atezolizumab for the treatment 
of patients with advanced NSCLC who have progressed after platinum-doublet chemotherapy.

\section{Durvalumab}

Durvalumab is an IgG1 monoclonal antibody which binds to PD-L1, thereby blocking and activating the PD-1 receptor on T-cells. The Fc region of durvalumab has been modified to stop it from inducing either antibody-dependent cytotoxicity (ADCC) or complement-dependent cytotoxicity (CDC).

ATLANTIC is a phase 2, open label, single arm trial in advanced NSCLC patients with $\geq 2$ lines of chemotherapy [23]. However, PD-L1 expression level cut off was $\geq 25 \%$ to enrol in the study. The study enrolled patients into 3 cohorts. Cohort 2 included patients who were either EGFR wild type or unknown status with either PD-L1 tumour expression of $\geq 25 \%$ or $\leq 25 \%$. Cohort 3 includes patients with at least $\geq 90 \%$ of PD-L1 tumour expression. The primary endpoint was ORR. The ORR in cohort 2 with $\geq 25 \%$ PD-L1 expression was $16.4 \%$ and $7.5 \%$ with $\leq 25 \%$ PD-L1 expression. The ORR in cohort 3 with $\geq 90 \%$ PD-L1 expression was $30.9 \%$. Durvalumab has also shown durable response particularly in cohort 3.

In a phase 3 MYSTIC trial, durvalumab alone or in combination with tremelimumab versus platinum doublets chemotherapy was tested in first line metastatic NSCLC. The primary endpoint was PFS and OS. Durvalumab alone or in combination with tremelimumab failed to meet the primary endpoints compared to standard chemotherapy. The results were announced in a press released without any other data.

\section{Avelumab}

Avelumab is a human IgG1 monoclonal antibody which blocks PD-L1 receptor. In a dose expansion phase 1 study, 184 patients with progressive or resistant advanced NS$\mathrm{CLC}$ were enrolled regardless of PD-L1 expression. The primary endpoint was to assess safety and tolerability. Overall, disease control rate was 50\%. The most common treatment related adverse effects were fatigue $(25 \%)$, infusion reaction (21\%), and nausea (13\%) [24]. Based on these results, avelumab moved into phase 3 trial (Javelin Lung 100) compared avelumab to platinum doublet chemotherapy as first line treatment of advanced PD-L1 positive NSCLC. The aim of the study is to show superiority of avelumab over platinum doublet chemotherapy. The trial is currently being recruited [25].

\section{Combination Therapy}

There has been great interest in using either PD-1 inhibitor or PD-L1 inhibitor as a backbone therapy given its durable response and favourable toxicity profiles in combination with chemotherapy, other novel immunotherapy, targeted therapy or radiotherapy. Many of these combinations are currently in early phase clinical trials (Table 2).

\section{Nivolumab + Platinum-doublet chemotherapy}

This phase 1 Checkmate 012 clinical trial has assessed the dose selection and toxicities of nivolumab in combination with platinum-based doublet chemotherapy in the first-line treatment of advanced NSCLC [26]. Fifty-six patients were enrolled into 1 of 3 cohorts. These cohorts were nivolumab $10 \mathrm{mg} / \mathrm{kg}$ with gemcitabine/ cisplatin or pemetrexed/cisplatin or nivolumab 5 or 10 $\mathrm{mg} / \mathrm{kg}$ plus paclitaxel/carboplatin based on the histology. Patients received 4 cycles of nivolumab plus chemotherapy then followed by maintenance nivolumab. The primary endpoint was safety and tolerability. The trial has recently reported a 3 years follow upwith ORR of $46 \%$ and $25 \%$ OS rate with median 19 months. The ORR and OS showed similar results regardless of PD-L1 expression. The duration of response was 10 months with median PFS of 6 months. However, this need to be validated further in phase 3 trial, Checkmate 227.

Table 2: Clinical trials assessing PD-1 or PD-L1 immune checkpoint inhibitors in combination with other therapy in NSCLC.

\begin{tabular}{|c|c|c|c|c|c|c|}
\hline Clinical Trials & $\begin{array}{l}\text { Lines of } \\
\text { Treatment }\end{array}$ & Phase & Sample Size & ORR (\%) & PFS (Months) & OS \\
\hline \multirow[t]{2}{*}{ Checkmate 012: Nivolumab + PT-DC } & 1 & One & 56 & $46 \%$ & 6 months & Median 19 months \\
\hline & & & & & & 2-year: $25 \%$ \\
\hline \multirow[t]{2}{*}{ Keynote 021: } & 1 & Two & & & & \\
\hline & & & & & & 18 months OS: \\
\hline Pembrolizumab + Carbo + Pem & & & & 57 & 19 & $70 \%$ \\
\hline Carbo + Pem & & & & 32 & 8.9 & $56 \%$ \\
\hline Atezolizumab + PT-DC & 1 & One & 37 & & Not Reported & Not Reported \\
\hline Atezolizumab + Carbo + Paclitaxel & & & 8 & 60 & & \\
\hline Atezolizumab + Carbo + Pemetrexed & & & 14 & 75 & & \\
\hline Atezolizumab + Carbo + Nab-paclitaxel & & & 15 & 62 & & \\
\hline \multirow[t]{2}{*}{ Checkmate 012: Nivo + Ipilimumab } & 1 & One & 77 & & & \\
\hline & & & & & 24 weeks (\%) & \\
\hline Nivo 3 mg/kg + Ipilimumab 1 mg/kg Q12w & & & & 47 & 68 & Not Reported \\
\hline Nivo 3 mg/kg + Ipilimumab 1 mg/kg Q6w & & & & 38 & 47 & Not Reported \\
\hline
\end{tabular}

ORR: Overall Response Rate; PFS: Progression Free Survival; OS: Overall Survival; AEs: Adverse Effects; PT-DC: Platinum-based doublet chemotherapy; Nivo: Nivolumab; Gem: Gemcitabine; Cis: Cisplatin; Pem: Pemetrexed; Carbo: Carboplatin; SqNSCLC: Squamous non-small cell lung cancer. 


\section{Pembrolizumab + Platinum-doublet chemotherapy}

Keynote-021 is an open-label phase 2 trial comparing platinum-doublet chemotherapy with or without pembrolizumab. Patients with advanced non-squamous NSCLC were randomly assigned into either 4 cycles of pembrolizumab $200 \mathrm{mg}$ plus carboplatin and pemetrexed every 3 weeks followed by pembrolizumab for 24 months and pemetrexed maintenance therapy or to 4 cycles of carboplatin and pemetrexed alone followed by maintenance pemetrexed. The primary end-point was ORR. An updated long-term results with median follow up of 18.7 months showed the ORR was $57 \%$ in the pembrolizumab plus chemotherapy arm and $32 \%$ in doublet chemotherapy arm with $p$ value 0.0029 . Median PFS was significantly longer with 19 months in the pembrolizumab plus chemotherapy arm compared to 8.9 months in doublet chemotherapy arm. 18 months OS was $70 \%$ in pembrolizumab plus chemotherapy compared to $56 \%$ in doublet chemotherapy. The most common grade $\geq 3$ treatment related toxicities were fatigue and neutropenia [27]. The combination of pembrolizumab and chemotherapy has recently been approved by the FDA for first-line treatment of metastatic non-squamous NSCLC.

\section{Atezolimumab + Platinum-doublet chemotherapy}

The preliminary report on the combination of PDL1 inhibitor with first line doublet chemotherapy was assessed in phase $1 \mathrm{~b}$. Patients with advanced NSCLC received atezolimumab (15 mg/kg IV every 3 weeks) plus carboplatin and paclitaxel, pemetrexed or weekly nab-paclitaxel for 4-6 cycles followed by maintenance atezolimumab until progression. The ORR was $60 \%$ to $75 \%$ across all arms independent of PD-L1 expression. There was no unexpected toxicity and the treatment was generally well tolerated [28]. A preliminary data from IMPower150, which is a phase 3, open label, randomised study of atezolizumab in combination with carboplatin and paclitaxel with or without bevacizumab compared with carboplatin and paclitaxel with bevacizumab in chemotherapy naïve stage 4 NSCLC [29]. The primary endpoints were PFS and OS. The PFS from the quadruple combination was slightly improved from triplet's combination from 6.8 months to 8.3 months. Similarly, the ORR was slightly improved from $48 \%$ to $64 \%$. The benefits were seen across all levels of PD-L1 expression, however greater with high PD-L1 expression. There is no new safety signals reported. The OS data remain immature at the time of report. Furthermore, there are no results available for atezolimumab plus chemotherapy without bevacizumab.

\section{Nivolumab + Ipilimumab}

The combination nivolumab with ipilimumab has shown to be safe and effective in enhancing T-cells in melanoma and has recently been approved by FDA. This combination of immunotherapy in NSCLC has now been validated in the phase 1 Checkmate 012 trial. This trial addressed the dosing and toxicity of nivolumab with ipilimumab in first-line advanced NSCLC. 77 patients were randomly allocated into either nivolumab $3 \mathrm{mg} /$ $\mathrm{kg}$ every 2 weeks plus ipilimumab $1 \mathrm{mg} / \mathrm{kg}$ every 12 weeks, or nivolumab $3 \mathrm{mg} / \mathrm{kg}$ every 2 weeks plus ipilimumab $1 \mathrm{mg} / \mathrm{kg}$ every 6 weeks. The primary endpoint was safety. Grade $\geq 3$ treatment related toxicities were similar in both groups with $37 \%$ in ipilimumab every 12 weeks arm and 33\% in every 6 weeks arm. This includes pneumonitis (5\%), adrenal insufficiency (3\%-5\%), and colitis (3\%-5\%). The ORR in nivolumab plus ipilimumab every 12 weeks, and nivolumab plus ipilimumab every 6 weeks, compared with nivolumab monotherapy were $47 \%, 38 \%$, and $23 \%$ respectively [ 30 ]. The ORR was also higher in patient with $\geq 50 \%$ PD-L1 expression from the combination arms. Compared to nivolumab monotherapy, the combination treatment showed higher response rate and durable. The combination therapy is now being evaluated in phase 3 trial, Checkmate-227, against standard of care.

\section{Future Direction}

The evolution of metastatic NSCLC treatment is changing rapidly with immune checkpoint inhibitors showing promising results particularly in patients without driver mutations. At present, there are clear indication to use single agent pembrolizumab as first-line treatment in metastatic NSCLC patient with high PD-L1 expression. Beyond this, data for various combinations immunotherapy with either chemotherapy, targeted therapy or other immunotherapeutic agent in upfront management of NSCLC remains immature. The results are encouraging but it need to show a significant survival benefits for practice to change. Clearly, there are strong indications for the use of PD-1 or PD-L1 inhibitors following failure of platinum-based doublet chemotherapy as compared to docetaxel.

Other essential questions remain unanswered including ways to select appropriate patients based on biomarkers for these immune checkpoint inhibitors. Despite its limitations, PD-L1 is the only biomarker used to guide immunotherapy currently. Nonetheless, increasing preliminary data are supporting the use of immunotherapy in PD-L1 negative disease especially when combining with other agents. These predictive biomarkers are currently being studied i.e. tumour mutation burden (TMB), circulating free DNA and CD8 tumour infiltrating lymphocytes may potentially help select appropriate immunotherapy agents for the right patients. The future of metastatic NSCLC management will likely be increasingly complex as we moves toward personalised medicine for individual pathology.

\section{References}

1. Ferlay J, Shin HR, Bray F, Forman D, Mathers C, et al. (2010) Estimates of worldwide burden of cancer in 2008: GLOBOCAN 2008. Int J Cancer 127: 2893-2917. 
2. Howlader N, Noone AM, Krapcho M, Neyman N, Aminou R, et al. (2012) SEER Cancer Statistics Review, 1975-2009 (Vintage 2009 Populations), National Cancer Institute.

3. Spiro SG, Rudd RM, Souhami RL, Brown J, Fairlamb DJ, et al. (2004) Chemotherapy versus supportive care in advanced non-small cell lung cancer: improved survival without detriment to quality of life. Thorax 59: 828-836.

4. Schiller $\mathrm{JH}$, Harrington $\mathrm{D}$, Belani $\mathrm{CP}$, Langer $\mathrm{C}$, Sandler $\mathrm{A}$, et al. (2002) Comparison of four chemotherapy regimens for advanced non-small-cell lung cancer. N Engl J Med 346: 92-98.

5. Scagliotti GV, Parikh P, Von Pawel J, Biesma B, Vansteenkiste J, et al. (2008) Phase III study comparing cisplatin plus gemcitabine with cisplatin plus pemetrexed in chemotherapy-naive patients with advanced-stage non-small-cell lung cancer. J Clin Oncol 26: 3543-3551.

6. Fife BT, Pauken KE (2011) The role of the PD-1 pathway in autoimmunity and peripheral tolerance. Ann N Y Acad Sci 1217: 45-59.

7. Herbst RS, Gordon MS, Fine GD, Sosman JA, Soria JC, et al. (2013) A study of MPDL3280A, an engineered PD$\mathrm{L} 1$ antibody in patients with locally advanced or metastatic tumors. J Clin Oncol 31.

8. Chen YB, Mu CY, Huang JA (2012) Clinical significance of programmed death-1 ligand-1 expression in patients with non-small cell lung cancer: a 5-year-follow-up study. Tumori 98: 751-755.

9. Pardoll DM (2012) The blockade of immune checkpoints in cancer immunotherapy. Nat Rev Cancer 12: 252-264.

10. Krummel MF, Allison JP (1995) CD28 and CTLA-4 have opposing effects on the response of $T$ cells to stimulation. $J$ Exp Med 182: 459-465.

11. Walunas TL, Bakker CY, Bluestone JA (1996) CTLA-4 ligation blocks CD28-dependent $T$ cell activation. J Exp Med 183: $2541-2550$.

12. Hedy L Kindler, Arnaud Scherpereel, Luana Calabrò, Joachim Aerts, Susana Cedres Perez, et al. (2016) Tremelimumab as second- or third-line treatment of unresectable malignant mesothelioma (MM): Results from the global, double-blind, placebo-controlled determine study. J Clin Oncol 34: 8502-8502.

13. Hodi FS, O'Day SJ, McDermott DF, Weber RW, Sosman JA, et al. (2010) Improved Survival with Ipilimumab in Patients with Metastatic Melanoma. N Engl J Med 363: 711-723.

14. Herbst RS, Baas P, Kim DW, Felip E, Perez-Gracia JL, et al. (2016) Pembrolizumab versus docetaxel for previously treated, PD-L1-positive, advanced non-small-cell lung cancer (KEYNOTE-010): a randomised controlled trial. Lancet 387: $1540-1550$.

15. Brahmer JR, Rodríguez-Abreu D, Robinson AG (2017) Updated analysis of KEYNOTE-024: pembrolizumab vs. platinum-based chemotherapy for advanced NSCLC with PD-L1 TPS $\geq 50 \%$.

16. Mok T, Wu YL, Sadowski S, Zhang J, Rangwala R, et al. (2016) Pembrolizumab (MK-3475) versus platinum-based chemotherapy for PD-L1+ NSCLC in a phase 3, randomized, open-label study: KEYNOTE-042. J Thorac Oncol 11: 142.

17. Brahmer J, Reckamp KL, Baas P, Crino L, Eberhardt WE, et al. (2015) Nivolumab versus docetaxel in advanced squamous-cell non-small-cell lung cancer. $\mathrm{N}$ Engl $\mathrm{J}$ Med 373: 123-135.

18. Borghaei H, Paz-Ares L, Horn L, Spigel DR, Steins M, et al. (2015) Nivolumab versus docetaxel in advanced non- squamous non-small-cell lung cancer. N Engl J Med 373: 1627-1639.

19. Socinski M, Creelan B, Horn L, Reck M, Paz-Ares L, et al. (2016) CheckMate 026: A phase 3 trial of nivolumab vs investigator's choice (IC) of platinum-based doublet chemotherapy (PT-DC) as first-line therapy for stage iv/recurrent programmed death ligand 1 (PD-L1)-positive NSCLC. Ann Oncol 27: LBA7 PR.

20. Besse B, Johnson M, Janne PA, Garassino M, Eberhardt WEE, et al. (2015) Phase II, single-arm trial (BIRCH) of atezolizumab as first-line or subsequent therapy for locally advanced or metastatic PD-L1-selected non-small cell lung cancer. 2015 European Cancer Congress.

21. Fehrenbacher $L$, Spira $A$, Ballinger $M$, Kowanetz $M$, Vansteenkiste $\mathrm{J}$, et al. (2016) Atezolizumab versus docetaxel for patients with previously treated non-small-cell lung cancer (POPLAR): A multicentre, open-label, phase 2 randomised controlled trial. Lancet 387: 1837-1846.

22. Rittmeyer A, Barlesi F, Waterkamp D, Park K, Ciardiello F, et al. (2017) Atezolizumab versus docetaxel in patients with previously treated non-small-cell lung cancer (OAK): a phase 3 , open-label, multicentre randomised controlled trial. Lancet 389: 255-265.

23. Garassino M, Vansteenkiste $\mathrm{J}$, Kim JH, Léna $\mathrm{H}$, Mazières $\mathrm{J}$, et al. (2017) Durvalumab in 3rd-Line Locally Advanced or Metastatic, EGFR/ALK Wild-Type NSCLC: Results from the Phase 2 ATLANTIC Study. J Thorac Oncol 12: S10-S11.

24. Gulley JL, Rajan A, Spigel DR, lannotti N, Chandler J, et al. (2017) Avelumab for patients with previously treated metastatic or recurrent non-small cell lung cancer (JAVELIN Solid Tumor): dose-expansion cohort of a multicentre, open label, phase $1 \mathrm{~b}$ trial. Lancet Oncol 18: 599-610.

25. Reck M, Yang CH, Postmus PE, Barlesi F, Font EF, et al. (2017) JAVELIN Lung 100: updated design of a phase 3 trial of avelumab vs platinum doublet chemotherapy as first line treatment for metastatic or recurrent PD-L1 non-small cell lung cancer (NSCLC). Annuals of Oncology 28: 460-496.

26. Juergens RA, Hellmann MD, Brahmer JR, Borghaei $H$, Gettinger S, et al. (2017) First line nivolumab plus platinum-based doublet chemotherapy for advanced NSCLC: Checkmate 0123 years-update. International Association for the Study of Lung cancer $18^{\text {th }}$ World Conference on Lung Cancer.

27. Borghaei H, Langer CJ, Gadgeel SM, Papadimitrakopoulou VA, Patnaik A, et al. (2017) Updated results from KEYNOTE-021 cohort G: A randomised, phase 2 study of pemetrexed and carboplatin (PC) with or without pembrolizumab (pembro) as first-line therapy for advanced nonsquamous NSCLC. Ann Oncol 28.

28. Liu SV, Powderly JD, Camidge DR, Ready N, Heist RS, et al. (2015) Safety and efficacy of MPDL3280A (anti-PDL1) in combination with platinum-based doublet chemotherapy in patients with advanced non-small cell lung cancer (NSCLC). J Clin Oncol 15: 8030-8030.

29. (2017) Primary PFS and safety analyses of a randomized phase III study of carboplatin + paclitaxel +/- bevacizumab, with or without atezolizumab in $1 \mathrm{~L}$ non-squamous metastatic NSCLC (IMpower150). ESMO Immuno-Oncology Congress. Geneva, Switzerland, 2017.

30. Hellmann MD, Gettinger SN, Goldman JW, Brahmer JR, Borghaei $\mathrm{H}$, et al. (2017) Nivolumab plus ipilimumab as first-line treatment for advanced non-small-cell lung cancer (Checkmate 012): results of an open-label,phase 1, multicohort study. Lancet Oncol 18: 31-41. 